\title{
Imaging in a rare case of neuroendocrine tumour with skin metastases
}

\author{
Marcin Białecki ${ }^{A, B, C, C, D, E, F}$, Agnieszka Białecka ${ }^{2 A, B, C, D, E, F}$, Kaja Męcińska-Jundziłł2A,B,C,D,E,F, Urszula Adamska ${ }^{2 A, B, C, D, E, F}$, \\ Anna Kasperska ${ }^{3 A, B, C, D, E, F}$, Rafał Czajkowski2A,B,C,D,E,F
}

'Department of Radiology and Diagnostic Imaging, Nicolaus Copernicus University in Toruń, Collegium Medicum in Bydgoszcz, Bydgoszcz, Poland ${ }^{2}$ Chair of Dermatology, Sexually Transmitted Diseases and Immunodermatology, Nicolaus Copernicus University in Toruń, Collegium Medicum in Bydgoszcz, Bydgoszcz, Poland

${ }^{3}$ Departament of Pathomorphology, Nicolaus Copernicus University in Toruń, Collegium Medicum in Bydgoszcz, Bydgoszcz, Poland

\section{Abstract}

Purpose: Disseminated malignancies are a diagnostic and therapeutic challenge that is often encountered in radiology. Finding the primary tumour is crucial for planning proper surgical and oncological treatment. Computed tomography (CT) of the thorax and abdomen is typically the initial examination. However, abdominal magnetic resonance imaging (MRI) or positron emission tomography (PET/CT) or PET/MRI are often subsequently performed. Histopathological examination of metastatic tumours is performed as well, followed by immunohistochemistry. The aim of the report was to present diagnostic workup in a rare case of skin metastases.

Case report: A 72-year-old patient was admitted to a dermatology ward because of skin lesions - violaceous nodules localised on the hair-covered skin of the head. On abdominal CT, a generalised neoplastic process with metastases in the liver, pancreas, adrenal glands, lymph nodes, bones, thoracic wall, and a suspected metastasis in the right breast was revealed. Histopathology of the skin nodules confirmed a neuroendocrine tumour. Metastases of a pancreatic neuroendocrine tumour or small-cell lung cancer were suspected on immunohistochemistry. The patient died before we were able to localise the primary source of the tumour and provide treatment.

Conclusions: Skin metastases are relatively rare, aggravate the prognosis, and usually indicate spread of the neoplastic process in the internal organs. It is not always possible to localise the primary tumour using radiological imaging. In such cases, co-operation with the pathologist is crucial as are the results of histopathological and immunohistochemical examinations.

Key words: multidetector computed tomography, pancreatic neoplasms, skin abnormalities.

\section{Introduction}

Disseminated malignancies are a diagnostic and therapeutic challenge that is often encountered in radiology. The localisation of the primary tumour is crucial for planning appropriate surgical and oncological treatment. Sometimes, standard imaging techniques cannot reveal the primary tumour. In such cases, co-operation with the pathologist is needed in order establish a diagnosis based on histopathological and immunohistochemical examinations.

\section{Case report}

A 72-year-old female patient, diagnosed with Binswanger's disease, with a history of tuberculosis, after surgery due to a compression fracture of the L1 vertebra was admitted to a dermatology ward because of skin lesions -

Correspondence address:

Marcin Białecki, Department of Radiology and Diagnostic Imaging, Nicolaus Copernicus University in Toruń, Collegium Medicum in Bydgoszzz,

9 Skłodowskiej-Curie St., 85-094 Bydgoszzz, Poland, e-mail: mbialecki@poczta.fm

Authors' contribution:

A Study design · B Data collection · C Statistical analysis · D Data interpretation - E Manuscript preparation · F Literature search · G Funds collection 

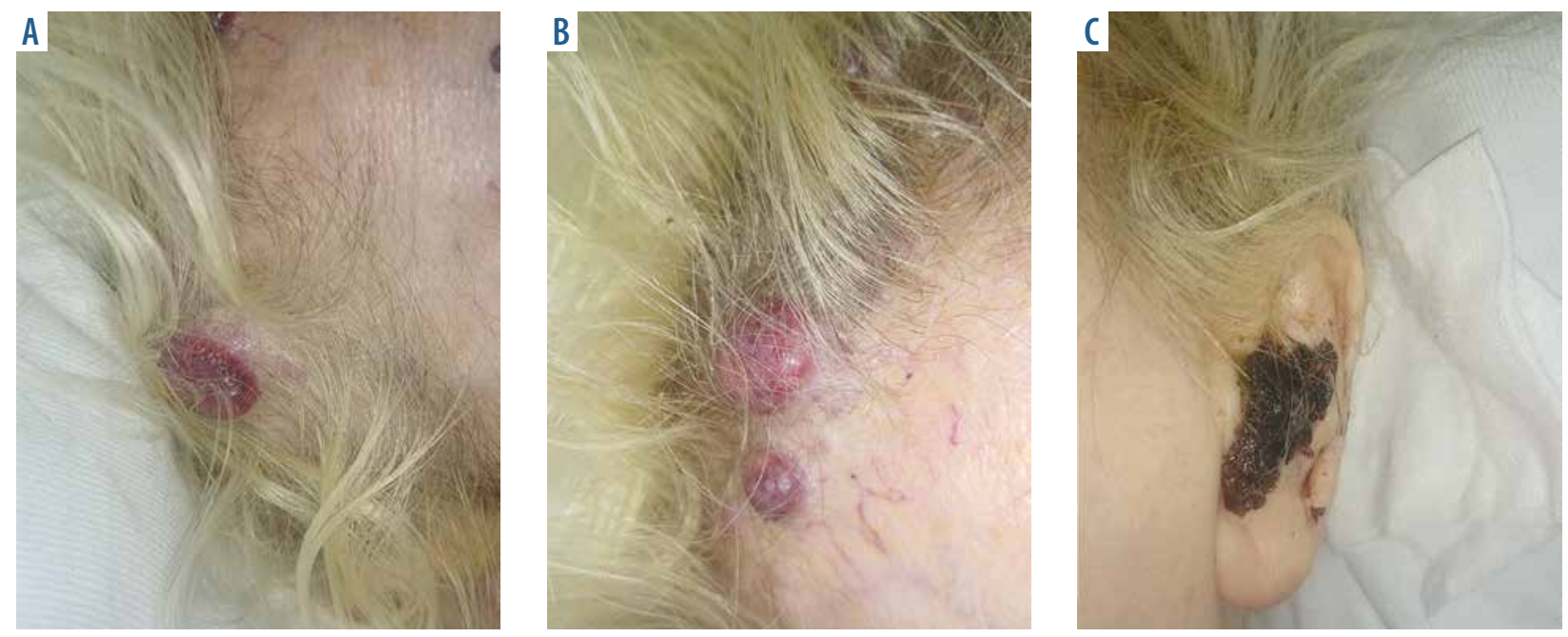

Figure 1. Skin metastasis. Skin of the head (A, B). Left external acoustic meatus (C)

violaceous noduleslocalised on the hair-covered skin of the head, in the left external acoustic meatus, on the back, and in the left groin (Figure 1). The first lesions appeared one month before admission, and had been rapidly progressing, engulfing the abovementioned body parts. Laboratory studies showed macrocytic anaemia, decreased activated partial thromboplastin time (APTT), a decreased level of folic acid, an increased level of D-dimer, and a slightly increased level of carcinoembryonic antigen (CEA). Symptomatic treatment was started - packed red blood cells were transfused and ionic balance was restored. Contrast-enhanced abdominal computed tomography (CT) revealed an enlargement of the head of pancreas and focal lesions with hypodense areas corresponding to necrotic changes. Evaluation of focal lesions in the liver and adrenal glands was impeded by artefacts resulting from L1 vertebroplasty. Two metastatic lesions were found in the liver, and similar lesions were also observed in both adrenal glands and in the wall of the tho- rax, destructing the ribs. Suspected metastatic lesions were found in the right breast (Figure 2). The clinical picture and imaging studies indicated a disseminated malignancy. Histopathology of the skin nodules confirmed a neuroendocrine tumour (Figure $3 \mathrm{~A}$ and $3 \mathrm{~B}$ ). Metastases of a pancreatic neuroendocrine tumour or small-cell lung cancer were suspected based on a positive cytokeratin reaction (CK Pan+, CK18+, CK19+, synaptophysin+) on immunohistochemistry (Figure 3C and 3D). Because of a poor general condition, the patient was transferred to an endocrinology ward, where she died before determining the primary tumour and did not receive treatment.

\section{Discussion}

Skin metastases are an important therapeutic issue although their occurrence is rare. Skin metastases most commonly occur in the course of breast cancer in women and lung cancer in men. They usually form violaceous nodules and
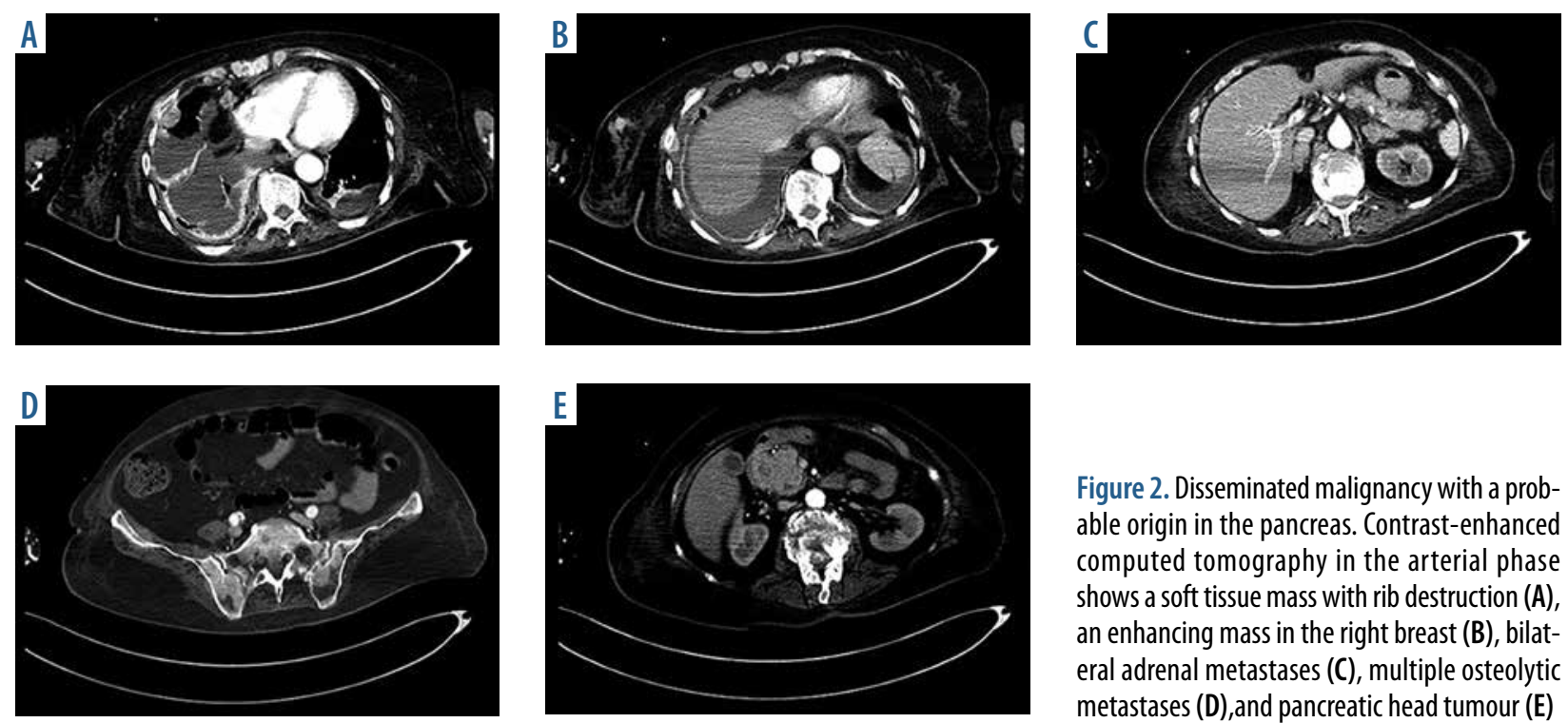

Figure 2. Disseminated malignancy with a probable origin in the pancreas. Contrast-enhanced computed tomography in the arterial phase shows a soft tissue mass with rib destruction (A), an enhancing mass in the right breast (B), bilateral adrenal metastases (C), multiple osteolytic metastases (D), and pancreatic head tumour (E) 

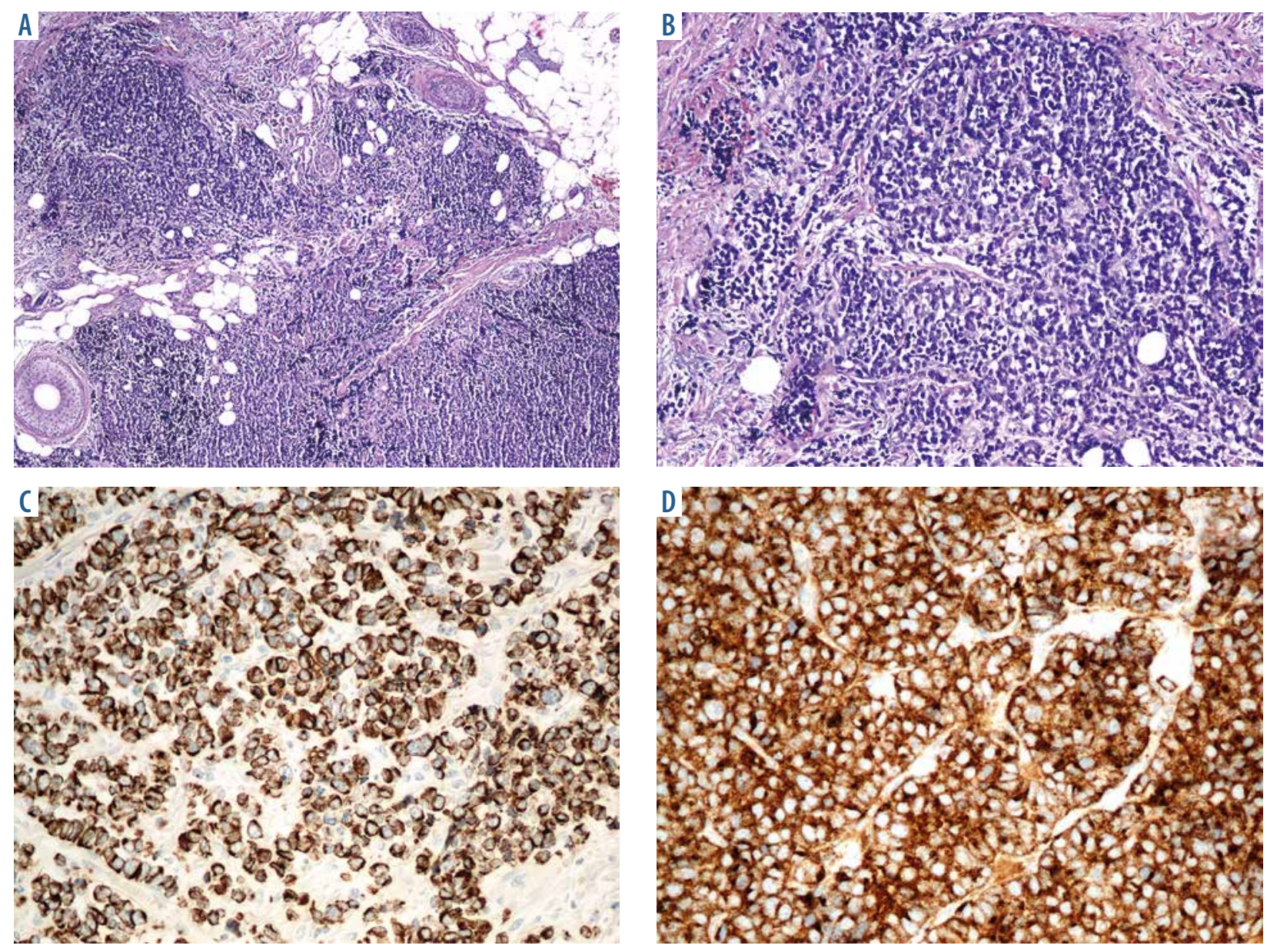

Figure 3. Histopathological examination, H and E. Cells are small, round, with the same appearance and rim of narrow cytoplasm. Round nuclei, "salt pepper" chromatin appearance with numerous nucleoli. Several cell division figures, including pathological mitosis. $\times 40$ (A). $\times 100$ (B). Immunohistochemistry $\times 200$. CK Pan + (C). Synaptophysin + (D)

tumours, sometimes decaying. These changes can most often be found on the skin of the head, torso, and less commonly of the limbs. Skin metastases appear in different locations, sometimes distant from the primary focus, and they usually do not cause pain [1]. Their appearance is usually a sign of disease progression, and indicates poor prognosis [2]. Metastases can also be a sign of tumour recurrence after successful treatment or be the first symptom of malignancy. Merkel-cell carcinoma, hemangioma, angiogranuloma, keloids, primary skin cancers including squamous cell carcinoma (SCC), melanoma, and cancers of the sweat glands or other appendages are included most commonly in the differential diagnosis. The characteristic clinical picture of skin lesions in the discussed patient suggested metastases and thus malignancy as the primary disease.

In our patient, the source of skin metastases was a neuroendocrine tumour (NET), which is exceptionally rare. Neuroendocrine tumours are a group of heterogeneous tumours which originate from cells of the neuroendocrine system, scattered within different organs, and usually form in the lungs, small intestine, and rectum $[3,4]$. These tumours are diagnosed in $2.5-5$ per 100,000 people per year, women and men are equally affected, usually in their sixties $[4,5]$. It is reported that $9 \%$ to $19 \%$ of patients with neuroendocrine tumours are also diagnosed with disseminated metastases of an unknown primary source [6].

Tumours of unknown primary location constitute a very heterogeneous group of diseases. In around 50\% of cases, numerous metastases are found at diagnosis; from the most to the least common: in the lymph nodes, lungs, bones, liver, pleural cavity, and brain [7]. Location of the primary tumour is usually determined by means of medical imaging. Typically, CT of the thorax or the abdomen is performed, and sometimes head CT is necessary. Although CT exposes patients to negative effects of ionising radiation, it is easy, fast, relatively cheap, and recommended by most oncological guidelines for determining the stage of disease. The diagnostic examination tools that are also used to determine the primary focus are magnetic resonance imaging (MRI) and scintigraphic tests, such as somatostatin receptor scintigraphy (SRS) [8]. However, MRI is expensive and not easily available in some facilities. On the other hand, the effectiveness of SRS examination used to locate the primary focus of a small intestine neuroendocrine tumour is estimated at only $37 \%$ [9].

Much attention has focused on the diagnosis and treatment of pancreatic endocrine tumours (PETs). On abdominal CT, these tumours can be seen as distinctive 
solid masses which tend to model surrounding structures. They are characterised by intense contrast enhancement in both the arterial and the venous phase, which is due to a large net of capillary vessels [10]. Smaller changes are usually more homogeneous, and bigger tumours are commonly characterised by heterogeneous enhancement with areas of cystic degeneration, necrosis, fibrosis, and calcification [11]. Sensitivity and specificity are high for CT- and MRI-assisted localisation of pancreatic neuroendocrine tumours, but detectability of these tumours by means of these techniques is much lower [12].

Neuroendocrine tumours can also be imaged with nuclear medicine techniques that utilise two types of radiolabelling, namely, receptor expression and metabolic activity of the tumour [13]. In 2017, a study showed that ${ }^{68} \mathrm{Ga}$-DOTA-TOC PET/CT using the ${ }^{68} \mathrm{Ga}$-DOTAD-Phe ${ }^{1}$-Try ${ }^{3}$-Octreotide label was helpful in localising primary neuroendocrine tumours in $37 \%$ of patients with disseminated neoplastic disease in whom conventional imaging techniques, such as CT or MRI, could not contribute to final diagnosis [8]. This examination is particularly useful in patients with an unknown primary focus in low-grade and intermediate-grade metastases. Our patient was diagnosed with high-grade metastases, thus an examination using ${ }^{68} \mathrm{Ga}$-DOTA-D-Phe ${ }^{1}$-Try ${ }^{3}$-Octreotide could not have been sufficient to establish a final diagnosis. Another study, performed in 2016, compared the effectiveness of PET/CT and PET/MRI in localising primary tumours in patients with metastatic neoplastic disease [14]. It showed that both hybrid imaging techniques have comparable diagnostic capabilities in diagnosing primary cancers and metastases, with PET/MRI providing the best results in the neck and $\mathrm{PET} / \mathrm{CT}$ in the lungs. As ionising radiation doses used in $\mathrm{PET} / \mathrm{MRI}$ are much lower, it can be an effective alternative to PET/CT, especially for treatment monitoring that requires repeat imaging [14].

Conventional ultrasound imaging is typically used as a screening test, and is sometimes followed by CT or MRI. Ultrasound guidance can be helpful in performing biopsy of lesions or metastatic lymph nodes as it can determine the place of specimen collection. Contrast-enhanced ultrasonography (CEUS) is a promising modality which uses intravenous contrasting media and measures contrast enhancement on ultrasound images [15]. In CT, iodinated contrast agents are typically used, which can lead to contrast-induced nephropathy (CIN), especially in elderly patients with chronic kidney disease or diabetes [16]. CEUS uses intravenous microbubbles, which allows to visualize local tissue vascularization and perfusion and is not associated with CIN [17].

Neuroendocrine tumours detected on imaging should be differentiated from other vessel-rich lesions, e.g. metastases, neurogenic tumours, vessel malformations, or primary exocrine tumours [18].

Radiological imaging is often unspecific, and neoplastic disease can be disseminated to such an extent that it is impossible to determine the primary focus by CT, MRI, SRS, or PET. In some cases, only co-operation between clinicians, radiologists, and pathologists can lead to final diagnosis and localisation of the primary tumour. The results of the histopathological and immunochemical examinations in our patient indicated a pancreatic tumour or small-cell lung cancer. In order to confirm the primary pancreatic tumour, additional immunohistochemical assays would have been necessary, namely: chromogranin A, Ki67/MIB1, hormone expression assay, and assessments determining the stage of expression and type of somatostatin receptors. In order to confirm a primary lung tumour, a cytological sputum examination, bronchial swab, and bronchoalveolar lavage would have been necessary. On immunohistochemistry, a positive result for TTF-1 would have confirmed primary lung cancer. These examinations were not conducted because of the patient's death. However, determining the source of a neoplastic disease in patients with neuroendocrine tumours seems to be crucial as even in patients with NET with non-resectable liver metastases localising the primary focus is beneficial [19].

\section{Conclusions}

Skin metastases are relatively rare, aggravate the prognosis, and usually indicate spread of the malignant process to the internal organs. Unspecific clinical picture can be diagnostically challenging. Imaging studies do not always indicate the localization of the primary tumour. However, they help determine the clinical advancement of the neoplastic disease. The location of the primary tumour should usually be confirmed by histopathology and immunohistochemistry.

\section{Conflict of interest}

The authors report no conflict of interest.

\section{References}

1. Kamińska-Winciorek G, Wydmański J, Januszewski K, et al. Dermoscopy of nodular skin metastases from the gastrointestinal primary cancer. Postepy Dermatol Alergol 2015; 32: 312-316.

2. Chauhan A, Sharma AK, Sunita BS. Cutaneous metastasis as primary presentation in unsuspected carcinoma esophagus: Report of two cases. J Cancer Res Ther 2015; 11: 667.
3. Hendifar AE, Marchevsky AM, Tuli R. Neuroendocrine tumors of the lung: current challenges and advances in the diagnosis and management of well-differentiated disease. J Thorac Oncol 2017; 12: 425-436.

4. Yao JC, Hassan M, Phan A, et al. One hundred years after "carcinoid": epidemiology of and prognostic factors for neuroendocrine tumors in 35,825 cases in the United States. J Clin Oncol 2008; 26: 3063-3072. 
5. Krawczyk J, Świeboda-Sadlej A. Guzy neuroendokrynne (NET) ze szczególnym uwzględnieniem guzów neuroendokrynnych przewodu pokarmowego (GEP-NET) - diagnostyka i leczenie. Medycyna po Dyplomie 2010; 19: 40-47 [In Polish].

6. Bellizzi AM. Assigning site of origin in metastatic neuroendocrine neoplasms: A clinically significant application of diagnostic immunohistochemistry. Adv Anat Pathol 2013; 20: 285-314.

7. Krzakowski M, Meder J, Olszewski W. Neoplasms of unknow primary site Recommendations of the Polish Union of Oncology. J Oncol 2003; 53: 660-665.

8. Menda Y, O’Dorisio TM, Howe J, et al. Localization of unknown primary site with $68 \mathrm{Ga}$-DOTATOC PET/CT in patients with metastatic neuroendocrine tumor. J Nucl Med 2017; 58: 1054-1057.

9. Maxwell JE, Sherman SK, Menda Y, et al. Limitations of somatostatin scintigraphy in primary small bowel neuroendocrine tumors. J Surg Res 2014; 190: 548-553.

10. Lewis RB, Lattin GE Jr., Paal E. Pancreatic endocrine tumors: Radiologic-clinicopathologic correlation. Radiographics 2010; 30: 1445-1464.

11. Buetow PC, Miller DL, Parrino TV, et al. Islet cell tumors of the pancreas: clinical, radiologic, and pathologic correlation in diagnosis and localization. Radiographics 1997; 17: 453-472.

12. Kartalis N, Mucelli RM, Sundin A. Recent developments in imaging of pancreatic neuroendocrine tumors. Ann Gastroenterol 2015; 28 : 193-202.
13. Rufini V, Baum RP, Castaldi P, et al. Role of PET/CT in the functional imaging of endocrine pancreatic tumors. Abdom Imaging 2012; 37: 1004-1020.

14. Ruhlmann V, Ruhlmann M, Bellendorf A, et al. Hybrid imaging for detection of carcinoma of unknown primary: A preliminary comparison trial of whole-body PET/MRI versus PET/CT. Eur J Radiol 2016; 85: 1941-1947.

15. Białecki M, Białecka A, Laskowska K, et al. Contrast-enhanced ultrasonography in Crohn's disease diagnostics. Hepatogastroenterology 2015; 62: 927-932.

16. Serafin Z, Karolkiewicz M, Gruszka M, et al. High incidence of nephropathy in neurosurgical patients after intra-arterial administration of low-osmolar and iso-osmolar contrast media. Acta Radiol 2011; 52: 422-429.

17. Serafin Z, Białecki M, Białecka A, et al. Contrast-enhanced ultrasound for detection of Crohn's disease activity: Systematic review and meta-analysis. J Crohns Colitis 2016; 10: 354-362.

18. Bhosale PR, Menias CO, Balachandran A, et al. Vascular pancreatic lesions: Spectrum of imaging findings of malignant masses and mimics with pathologic correlation. Abdom Imaging 2013; 38: 802-817.

19. Capurso G, Rinzivillo M, Bettini R, et al. Systematic review of resection of primary midgut carcinoid tumour in patients with unresectable liver metastases. Br J Surg 2012; 99: 1480-1486. 